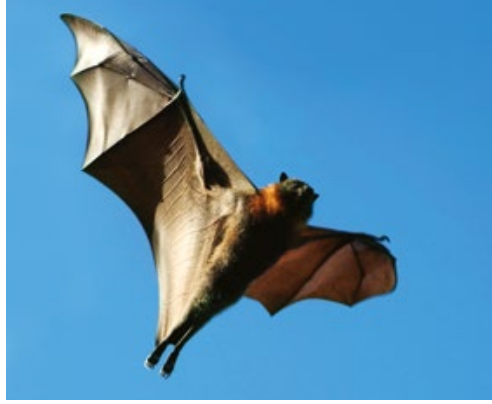

\section{CPD}

Tony Merritt, Kathryn Taylor, Keren Cox-Witton, Hume Field, Kate Wingett, Diana Mendez, Michelle Power, David Durrheim

\section{Background}

Australian bat lyssavirus (ABLV) causes human illness that is indistinguishable from classical rabies. All Australian bats have the potential to carry and transmit $A B L V$, and potentially risky human exposures to bats are common. ABLV infection has resulted in three human deaths in Australia since 1996.

\section{Objective}

The aim of this article is to equip general practitioners (GPs) to assist in the prevention and management of potential ABLV exposures in Australia, including complex clinical scenarios that are not fully addressed in current national guidelines.

\section{Discussion}

Potential ABLV exposures are frequently encountered in general practice. GPs play a critical role in risk mitigation for groups such as veterinarians and wildlife carers, and in triggering urgent multidisciplinary responses to potential exposures. Timely notification of the public health unit following a potential exposure is crucial to ensure appropriate assessment and access to correct treatment. Complex exposure scenarios require careful consideration.

\title{
Australian bat lyssavirus
}

AUStRALIAN BAt LySSAVIRUS (ABLV)

is regarded as endemic in Australian bats. It is one of 14 known lyssavirus species ${ }^{1}$ and causes human illness that is indistinguishable from classical rabies. It has resulted in three known human deaths in Australia since $1996 .^{2}$

All Australian bats have the potential to carry and transmit ABLV, and potentially risky human exposures to bats are common. Timely post-exposure prophylaxis (PEP) is critical to prevent ABLV disease.

\section{ABLV in bats}

ABLV was first identified in an encephalitic black flying fox in Queensland, Australia, in 1996. ${ }^{3}$ Subsequently, ABLV has been identified in all four mainland species of flying fox and one species of insectivorous microbat, with serological evidence of ABLV exposure in a range of microbat species. ${ }^{4}$ The overall prevalence of ABLV in bats is estimated to be $<1 \%$ in healthy bats, and 5-10\% in sick, injured or orphaned bats. ${ }^{4,5}$

Clinical signs of ABLV infection in bats include aggression, paralysis, respiratory difficulties, unusual vocalisation, inability to fly, tremors and convulsions. However, infected bats may appear healthy and behave normally.

\section{Transmission from bats}

The greatest risk for ABLV transmission from infected bats to people is by direct inoculation of saliva through a bite or scratch that breaks the skin. Bat saliva in direct contact with mucosal surfaces (ie nose, eyes or mouth) also poses a potential transmission risk. Human exposure to bats is common and increasing, with $>100$ residents requiring PEP each year in New South Wales alone. ${ }^{6}$ Typical scenarios include unvaccinated civilians attempting to rescue injured or sick bats, or children whose behaviour puts them at risk. Exposure to bat blood, urine or faeces is not considered an infection risk for ABLV.

ABLV infection was documented in two horses in Australia in 2013. ${ }^{7}$ Apart from bats, humans and horses, naturally occurring ABLV infection has not been documented in any other animal species (eg dogs, cats).

\section{Clinical features}

Three human deaths resulting from ABLV infection have been identified in Australia, occurring in 1996, 1998 and 2013. ${ }^{2}$ Transmission from flying foxes and an insectivorous microbat have been implicated, ${ }^{8-10}$ and all three cases displayed features of encephalitic (furious) rabies.

The incubation period for ABLV is likely to be similar to that of rabies, usually three to eight weeks, but potentially as short as a few days or as long as several years. ${ }^{11}$ Extensive exposure wounds close to the central nervous system (CNS) (eg injuries to the head and neck) or to richly innervated areas (eg fingers), carry increased infection risk and may result in a shorter incubation period.

In furious rabies, prodromal symptoms may precede sensorineural dysfunction, which deteriorates into hyperactivity, aerophobia and/or hydrophobia, followed 
by convulsions. The clinical course following symptom onset is usually rapid, almost invariably progressing to death within a few days.

\section{Management of potential ABLV exposures}

Post-exposure management is recommended for a person with 'any bite or scratch from, or mucous membrane or broken skin contact with the saliva or neural tissues of, a bat in Australia or elsewhere in the world'. ${ }^{2}$

The key management elements are wound care and PEP with human rabies immunoglobulin (HRIG) and/or rabies vaccine. The appropriate management for each patient is determined by the nature of the exposure, rabies vaccination history and the patient's immune status. ${ }^{2}$ Case management should be assessed in collaboration with the local public health unit, which will also arrange supply of HRIG and vaccine at no cost to the patient where an exposure is considered to pose a significant threat for lyssavirus transmission. For example, a witnessed bat bite with a visible wound clearly warrants PEP. However, general practitioners (GPs) may be confronted with more complex exposure scenarios and a series of potentially challenging situations are discussed in this article.

\section{Wound care}

Every effort should be made to neutralise or inactivate the virus while it is still in the exposure wound and before it enters into the nervous system, where it is relatively protected. ${ }^{12}$ Wounds should be immediately and vigorously cleaned with soap and water for at least five minutes, then an iodine-containing antiseptic should be applied. Animal studies demonstrate substantial reduction in infection risk with thorough wound cleaning..$^{13}$ Tetanus and other wound infections should be considered, and primary suture should be avoided if possible.

\section{Human rabies immunoglobulin}

HRIG provides localised rabies antibody protection while awaiting a vaccine- induced response. HRIG dosing is based on the patient's weight. If HRIG is indicated, as much of the calculated dose should be injected into and around the exposure wound as possible, including into fingers and toes. If wounds are extensive, the HRIG should be diluted with normal saline to ensure the entire area is injected. Any remaining HRIG can be given via intramuscular injection at a site away from vaccine administration.

\section{Rabies vaccine}

For patients who are immunocompetent with no previous rabies vaccinations, four vaccine doses should be given (days 0, 3, 7 and 14). Patients who are immunocompromised require five doses (days o, 3, 7, 14 and 28) and subsequent confirmation of an adequate antibody response. Patients who were previously vaccinated should have two doses of vaccine (days o and 3), and do not require HRIG.

If the implicated bat is available and can be tested without putting others at risk, PEP can be modified. If bat test results can be available within 48 hours of exposure, HRIG and vaccination can be deferred until that time. ${ }^{2}$ If PEP has commenced, it may be discontinued if the bat tests negative for ABLV. The public health unit can advise on arrangements for bat testing.

PEP remains indicated for at-risk exposures to bats regardless of any delay in reporting.

\section{Complex ABLV exposure scenarios}

CASE 1. UNCERTAIN BAT EXPOSURE

A father presents with his son, two years of age. This morning, a live microbat was found on the curtains of the boy's bedroom, where he sleeps alone. There is no visible evidence of a bat bite or scratch on the child, but the father is concerned after reading that US authorities would consider rabies PEP in such a situation. The bat is not available for testing.

The Australian immunisation handbook notes that 'a person with an intellectual disability, an intoxicated person, a child, or a person who has been sleeping in a confined space with a bat present' may be 'unaware or unable to communicate that an exposure has occurred', and recommends contact with the relevant state or territory authority. ${ }^{14}$ Importantly, microbat bite marks may be so minor that the absence of visible wounds does not preclude an at-risk exposure. PEP should be considered in these circumstances. ${ }^{14}$

A small number of patients in North America have developed rabies through bat lyssavirus infection attributed to undocumented indoor exposure to microbats, and US guidance is readily found by patients on the internet. ${ }^{15}$ However, following a review in 2009, Canada no longer recommends vaccination when there is no evidence of direct contact with a bat. ${ }^{16}$

To date, there is no record of cryptic transmission of ABLV from Australian microbats to humans, despite the not uncommon presence of microbats in houses. While the risk of transmission from undocumented microbat exposure in Australia must be extremely low, a risk assessment should be made in consultation with an expert. If the bat can be safely submitted for testing, this approach is preferable given the very low potential risk. PEP can be delayed while awaiting laboratory results.

Recommendation: This scenario should be discussed with the public health unit. The transmission risk is extremely low but PEP will be considered on a caseby-case basis.

\section{CASE 2. A DEAD BAT}

A mother presents with her toddler daughter. A dead, squashed and smelly microbat was found inside the foot section of a one-piece sleeping garment this morning. The child had been walking on the bat before it was discovered, and it was unclear when the bat had entered the garment. There was no mark on the child's foot. The family were aware of microbats in the area but they had not seen any in the house. 
Australian guidelines now recommend that a risk assessment should be conducted for potential ABLV exposures to live and dead animals..$^{14}$ Environmental persistence of ABLV, based on research on classic rabies virus, is extremely limited. It is rapidly inactivated by heat and sunlight. ${ }^{2}$ Persistence of viable virus in a dead bat will also be limited, but the likely infective period is not clear.

Recommendation: This scenario should be discussed with the public health unit. Given the possibility that this child was exposed to the microbat while it was still alive or soon after, PEP including HRIG into the child's foot is likely to be appropriate.

\section{CASE 3. OTHER BAT FLUIDS}

A farmer reports being sprayed with a fine fluid mist when walking under roosting bats. This fluid may have entered his eyes and mouth. He is concerned that this could be bat saliva and worries that he is at risk of $A B L V$ infection.

Exposure to a fluid mist or droplets under roosting bats is not uncommon. This fluid is bat urine, not saliva, and such exposures pose no known risk for ABLV infection.

Exposure to bat saliva directly contacting mucosa, such as the eye, nose or mouth, is very uncommon and should prompt review with the public health unit.

Recommendation: This patient can be reassured that he is not at risk of ABLV infection from this exposure and no PEP is required.

\section{CASE 4. DOMESTIC PETS EXPOSED TO BATS}

A retiree presents after being bitten by his pet dog. Two weeks earlier, he had found his dog with a dead bat in its mouth. He could not identify any wounds to the dog at that time and did not take the dog to the vet. The dog had since been behaving normally but bit him when he attempted to remove some dog bones yesterday.

Naturally occurring ABLV infection has not been documented in any dog or cat in Australia, despite numerous bat encounters. However, seroconversion to ABLV has been documented following experimental inoculation of dogs and cats, ${ }^{17}$ and natural infection with ABLV has occurred in Australian horses. ${ }^{7}$ Infection with related bat lyssaviruses has been occasionally documented in terrestrial mammals on other continents. It is thus feasible that domestic pets could become infected following bat exposures. Following contact between a pet and a bat, veterinary advice should be sought from a private veterinarian or government veterinary officer..$^{18}$

Transmission from an infected pet to a human is theoretically possible, but the likelihood is very low. There is no documented evidence of bat lyssavirus transmission from an infected terrestrial animal to a human anywhere in world.

Any bite or scratch from a pet that is manifesting clinical signs or behavioural changes that are consistent with lyssavirus infection should prompt urgent contact with the public health unit. While variable and sometimes subtle, clinical signs of lyssavirus infection in animals may include excitation, paralysis, loss of normal social and behavioural responses, and unusual vocalisation. ${ }^{18}$ PEP has been administered in these circumstances on a very few occasions in Australia.

Recommendation: This scenario should be discussed with the public health unit and relevant veterinary authorities. If the dog's behaviour is assessed as not being suggestive of lyssavirus infection, no PEP would be indicated for the patient. Tetanus and other wound infections should be considered. The patient should consult a veterinarian regarding the dog's health and history of contact with a bat.

CASE 5. REPEATED EXPOSURES IN A VACCINATED BAT HANDLER

A volunteer wildlife carer has seen you on multiple occasions following bat bites and scratches. She received a three-dose pre-exposure course of rabies vaccines 10 years ago, and has subsequently had three courses of PEP rabies vaccine (nine doses in total). The last of these was given three years ago, and her viral neutralising antibody (VNAb) titre was $1.7 \mathrm{IU} / \mathrm{mL}$ when checked eight months ago. She reports a new bite to her left thumb from a flying fox that she is caring for. She does not want to have the bat euthanised for ABLV testing.

Pre-exposure rabies vaccination is recommended for bat handlers, veterinarians, wildlife officers and carers, and others who expect to come into direct contact with bats; and for laboratory personnel working with live lyssavirus. ${ }^{2}$ Only appropriately trained and vaccinated persons, using appropriate protective equipment, should handle bats. ${ }^{2}$ Close contact with bats anywhere in the world should be avoided by other community members.

Those with ongoing exposure risk (eg wildlife officers), should have regular serology: every two years for veterinarians and bat handlers, and six-monthly for laboratory personnel. A single booster dose of rabies vaccine is recommended if viral neutralising antibodies are below $0.5 \mathrm{IU} / \mathrm{mL}$ at the time of serological review.

Subsequent at-risk exposures do not require HRIG, but Australian guidelines conservatively recommend two further doses of vaccine regardless of serological status or repeated previous vaccinations. ${ }^{14}$

Recommendation: This scenario should be discussed with the public health unit. This patient should have a further two doses of rabies vaccine and the use of appropriate personal protective equipment when handling bats discussed. HRIG is not indicated.

\section{CASE 6. BAT EXPOSURE IN THE DISTANT PAST}

A teenager presents after reading media coverage about the risk of rabies following bat exposures and asks whether she needs any treatment. She was scratched on the forearm while rescuing a flying fox from a fence about 12 months earlier. The wound has healed, leaving a fine scar. She has not previously sought medical advice for this injury and has never had any rabies vaccinations. She is immunocompetent. 
While clinical disease typically develops three to eight weeks following exposure, the incubation period may be as long as several years, and PEP is indicated regardless of the time since the at-risk exposure.

Recommendation: This scenario should be discussed with the public health unit. This patient should receive HRIG injected into the site of the scratch and four doses of rabies vaccine.

\section{Conclusions}

Potential ABLV exposures are frequently encountered in general practice. GPs have a critical role in risk mitigation for groups such as veterinarians and wildlife carers, and in triggering an urgent multidisciplinary response to exposures. Timely notification of the public health unit following a potential exposure is crucial, to ensure appropriate assessment and access to correct treatment. Complex exposure scenarios require careful consideration.

\section{Key points}

- All bat exposures (ie bite, scratch, mucosal exposure to bat saliva) in Australia require urgent assessment and consideration for ABLV PEP.

- Exposure sites should immediately be cleaned vigorously with soap and water for at least five minutes, then an iodinecontaining antiseptic applied.

- Requirement for HRIG and rabies vaccine should be urgently assessed in conjunction with the public health unit, which will also arrange for supply of PEP.

- If HRIG is administered, the maximum dose possible should be injected in and around the exposure site.

- The bat should be submitted for testing if possible and if this can be done without placing others at risk of exposure.

- Only appropriately trained and vaccinated persons using appropriate protective equipment should handle bats.

\section{Authors}

Tony Merritt MBBS, MPH\&TM, FRACGP, FAFPHM, Public Health Physician, Hunter New England Population Health, NSW. Tony.merritt@hneahealth. nsw.gov.au
Kathryn Taylor BMed, MPH\&TM, FRACGP, FAFPHM, Public Health Physician, Hunter New England Population Health, NSW

Keren Cox-Witton BVSc, GradDipApplSc, Senior Project Officer, Wildlife Health Australia, NSW

Hume Field BVSc, MSc, PhD, MACVS, Science \& Policy Adviser, EcoHealth Alliance; Honorary Professor, School of Veterinary Science, University of Queensland, Qld

Kate Wingett BVSc (Hons), Veterinary Officer, Animal Biosecurity Services and Response, NSW Department of Primary Industries, NSW

Diana Mendez BVSc, MPH, PhD, Mentor and Postgraduate Research Methodology Lecturer, Cohort Doctoral Studies Program, College of Public

Health, Medical and Veterinary Sciences, James Cook University, Qld

Michelle Power BSc, PhD, Department of Biological Sciences, Macquarie University, NSW

David Durrheim MBChB, DrPH, FAFPHM, Public Health Physician, Hunter New England Population Health, NSW

Competing interests: None.

Provenance and peer review: Commissioned, externally peer reviewed.

\section{Acknowledgements}

The authors belong to The Regional One Health Partnership, which operates in New South Wales and Queensland, and brings together specialists from human, animal and environmental health.

\section{References}

1. International Committee on Taxonomy of Viruses. Virus taxonomy: 2016 release. ICTV, 2017. Available at https://talk.ictvonline.org/taxonomy [Accessed 18 August 2017].

2. Communicable Diseases Network Australia. Rabies virus and other lyssavirus (including Australian bat lyssavirus) exposures and infections. Canberra: CDNA, 2013. Available at www.health. gov.au/internet/main/publishing.nsf/Content/ ED62D139B56F7B80CA257BF0001B7422/\$File/ ABLV-rabies-song-gl-June2014.pdf [Accessed 18 August 2017].

3. Fraser GC, Hooper PT, Lunt RA, et al. Encephalitis caused by a Lyssavirus in fruit bats in Australia. Emerg Infect Dis 1996;2(4):327-31.

4. Field $\mathrm{H}$. The ecology of Hendra virus and Australian bat lyssavirus [PhD]. Brisbane: University of Queensland, 2005.

5. Barrett J. Australian bat lyssavirus [PhD]. Brisbane: University of Queensland, 2004

6. Kardamanidis K, Cashman P, Durrheim D. Travel and non-travel associated rabies post exposure treatment in New South Wales residents, Australia, 2007-2011: A cross-sectional analysis. Travel Med Infect Dis 2013;11(6):421-26.

7. Annand EJ, Reid PA. Clinical review of two fatal equine cases of infection with the insectivorous bat strain of Australian bat lyssavirus. Aust Vet J 2014;92(9):324-32.

8. Allworth A, Murray K, Morgan J. A human case of encephalitis due to a lyssavirus recently identified in fruit bats. Commun Dis Intell 1996;20(24):504.

9. Hanna JN, Carney I, Smith G, et al. Australian bat lyssavirus infection: A second human case, with a long incubation period. Med J Aust 2000;172(12):597-99.

10. Francis J, Nourse C, Vaska VL, et al. Australian bat lyssavirus in a child: The first reported case. Paediatrics 2014;133(4):e1063-67.
11. Heymann DL, editor. Control of communicable diseases manual. 20th edn. Washington: American Public Health Association, 2015.

12. Warrell MJ, Warrell DA. Rabies and other lyssavirus diseases. Lancet 2004;363:959-69.

13. Kaplan M, Cohen D, Koprowski H, Dean D, Ferrigan L. Studies on the local treatment of wounds for the prevention of rabies. Bull World Health Organ 1962;26:765-75.

14. Department of Health. The Australian immunisation handbook. 10th edn. Canberra: $\mathrm{DoH}, 2013$. Available at www.immunise.health.gov. au/internet/immunise/publishing.nsf/Content/ Handbook10-home handbook10part4 handb ook10-4-16 [Accessed 18 August 2017].

15. Centers for Disease Control and Prevention (CDC) Human rabies prevention - United States, 2008. Recommendations of the Advisory Committee on Immunization Practices. MMWR Recomm Rep 2008;57(RR03):1-26.

16. National Advisory Committee on Immunisation. Recommendations regarding the management of bat exposures to prevent human rabies. Canada Communicable Disease Report 2009;35(ACS 7):1-28.

17. McColl K, Chamberlain T, Lunt R, Newberry $K$ Westbury H. Susceptibility of domestic dogs and cats to Australian bat lyssavirus (ABLV). Vet Microbiol 2007;123(1-3):15-25.

18. Animal Health Australia. Disease strategy: Australian bat lyssavirus (Version 3.0). Australian Veterinary Emergency Plan (AUSVETPLAN), Edition 3. Canberra, ACT: Primary Industries Ministerial Council, 2009 